\title{
VALIDATION OF COUPLED SIMULATION FOR FIRE-EXPOSED STRUCTURES
}

\author{
S. Šulc*, V. Šmilauer**, L. Blesák, K. Cábová, F. Zeman, F. Wald ${ }^{* * *}$
}

\begin{abstract}
Structural fire assessment creates essential part in design and maintenance of steel, concrete or timber structures. Expensive empirical tests carried out in furnaces with gas burners present traditional approach. This article presents validation of linked computational approach for fire simulation and its effects on structure using adiabatic surface temperature approach. The simulation solves a weakly-linked problem, consisting of computational fluid dynamics (CFD), heat transfer and mechanical model. The temperature field from the CFD creates Cauchy and radiative boundary conditions for the thermal model. The temperature field from an element is passed further to the mechanical model, which induces thermal strains and modifies material parameters. This article presents a validation of the linked simulation, based on experiment with a timber beam exposed to fire in a furnace. The computation uses standard material properties given in EN 1995-1-2.
\end{abstract}

Keywords: thermo-mechanical model, virtual furnace, computational fluid dynamics, multiphysics, fire resistance

\section{Introduction}

Numerical models offer solution in both domains of fire evolution and structural behavior. Computational fluid dynamics (CFD) brings solution of Navier-Stokes equations, supplemented often with mass and species transport.

Weak and discretized form of equilibrium equations provides the framework for structural mechanical analysis in the form of finite element method (FEM). It allows accommodating non-linear material stress-strain laws, finite strains, changes of boundary conditions, temperature-dependent material properties, etc. Similarly, time and space discretization of non-stationary heat balance equation leads to solution of thermal problems. Today, thermo-mechanical simulations are well established in the majority of finite element codes using a staggered solution strategy.

In our computations CFD model uses Fire Dynamics Simulator (FDS) code, see NIST (2018), and the thermo-mechanical task runs in OOFEM code, see Patzák (2018). Both codes are interconnected with MuPIF's API python library, see MuPIF (2018), which transfers simulation data, exports them to VTK data formats, and orchestrates simulation runs. The software tool itself has been described, see Šulc (2017).

\section{Validation}

In this section we present a validation of the developed software tool against experiment. The subject of validation was thermal response of a timber beam exposed to fire in a furnace. As our FDS computations have already been validated by Cábová (2017), we focus on the linking with the thermal task.

Ing. Stanislav Šulc: Department of Mechanics, Faculty of Civil Engineering, Czech Technical University in Prague; Thákurova 7/2077; 166 29, Prague; CZ, stanislav.sulc@fsv.cvut.cz

** Doc. Ing. Vít Šmilauer, Ph.D.: Department of Mechanics, Faculty of Civil Engineering, Czech Technical University in Prague; Thákurova 7/2077; 166 29, Prague; CZ, vit.smilauer@fsv.cvut.cz

*** Prof. Ing. František Wald, CSc.: Department of Steel and Timber Structures, Faculty of Civil Engineering, Czech Technical University in Prague; Thákurova 7/2077; 166 29, Prague; CZ, wald@fsv.cvut.cz 


\subsection{Geometry and description of the task}

The furnace dimensions are: length $=4.00 \mathrm{~m}$, width $=2.95 \mathrm{~m}$ and height $=2.57 \mathrm{~m}$. See the horizontal and vertical sections in Figs. 1 and 2. Just under the ceiling were placed three glued timber beams, the beam cross section is in Fig. 3. Initial temperature was $10^{\circ} \mathrm{C}$. The maximum output of the burners was 258 $\mathrm{kw}$, the function of the output dependent on time is displayed in Fig. 6. The experiment lasted 30 minutes.

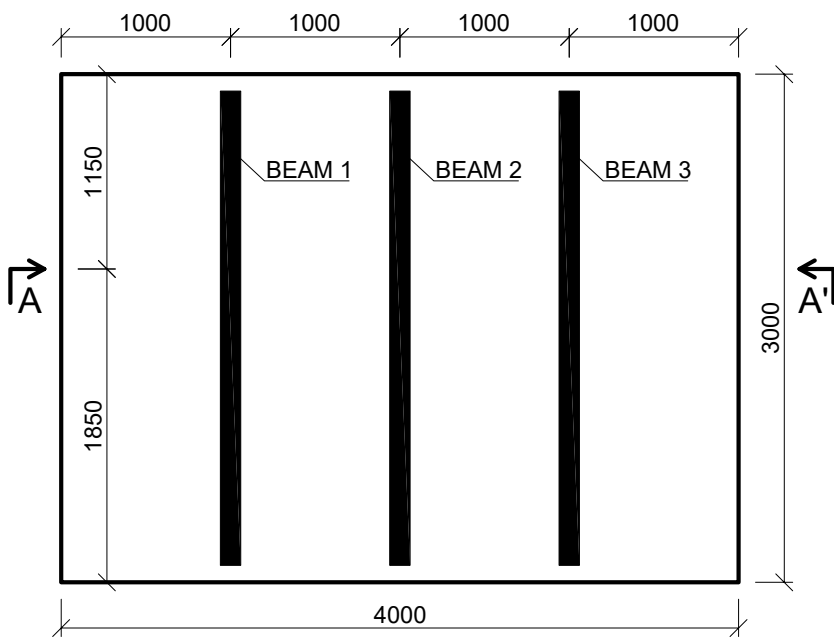

Fig. 1: Horizontal section of the furnace.

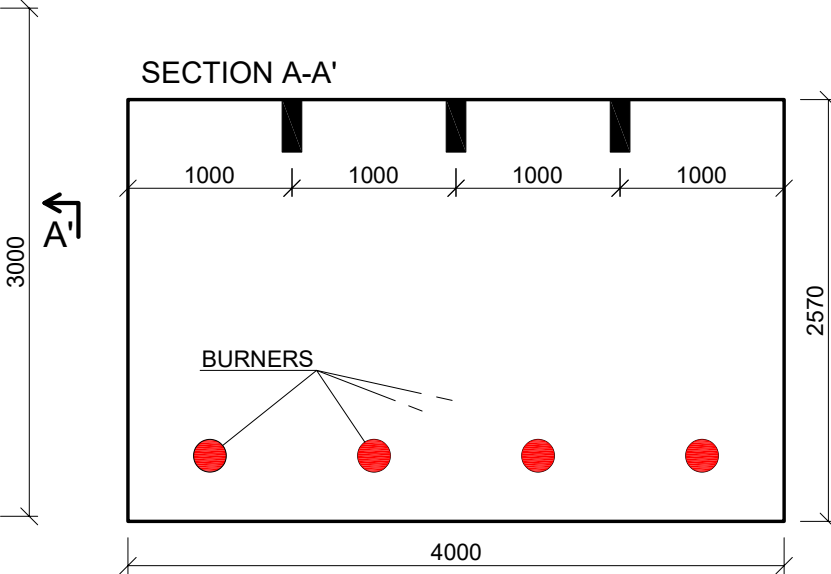

Fig. 2: Vertical section of the furnace.

The emissivity of timber surface was set to 0.95 , assuming carbonized surface. We found out that the value of heat transfer coefficient has almost no impact on the temperatures of the beam, thus we considered zero value.

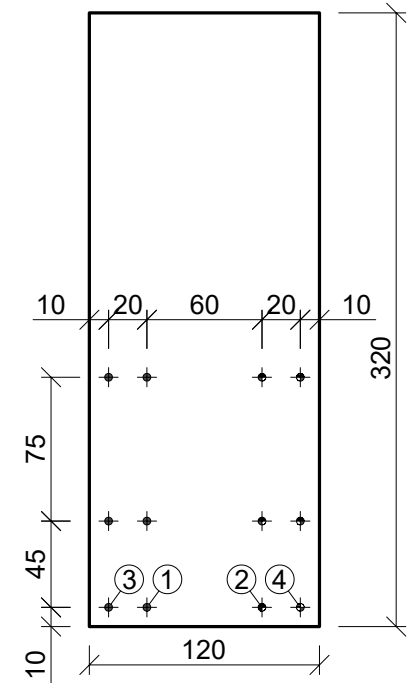

Fig. 3: Cross-section of the beam 3.

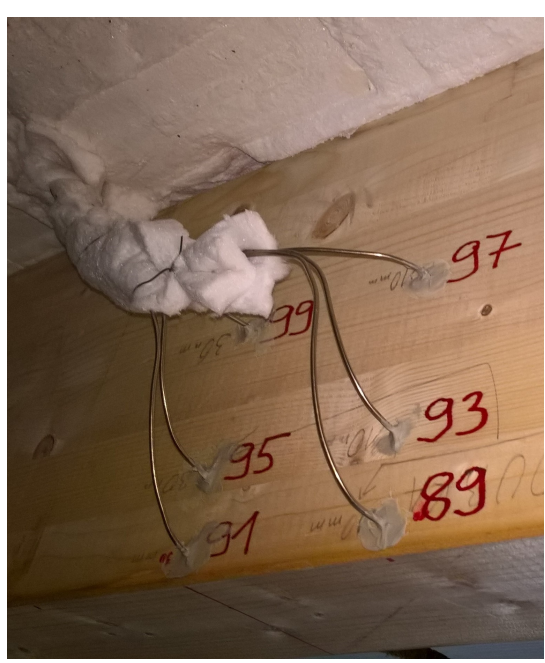

Fig. 4: Wiring to the sensors.

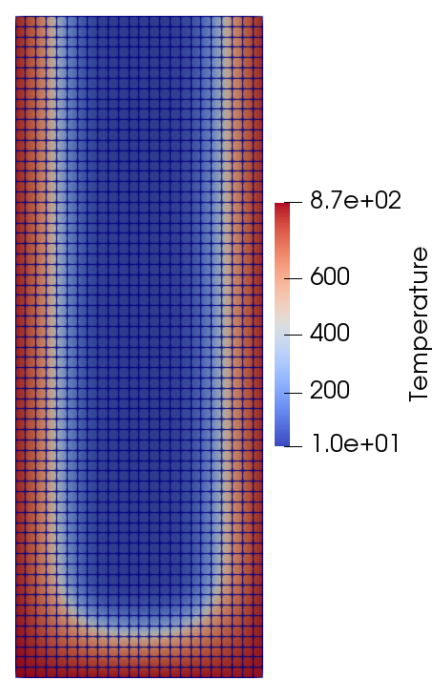

Fig. 5: Temperature in the crosssection of the beam in time $t=1800 \mathrm{~s}$.

\subsection{Material model}

We wanted to validate a response computed with standard material model, thus the material properties are taken from EN 1995-1-2, which takes into account the first 30 minutes of the flaring process. This model does not involve explicit burnout of timber, because it is taken into account in the parameters.

In Fig. 9 is displayed the thermal conductivity. Fig. 7 presents the timber density, which supposes initial moisture content of $13.5 \%$ and Fig. 8 presents the timber capacity, which involves a big peak due to the water evaporation around $100{ }^{\circ} \mathrm{C}$. As we can see in these figures, we modified the functions to be more suitable for a stable FEM computation, but the involved energy stays equal. 


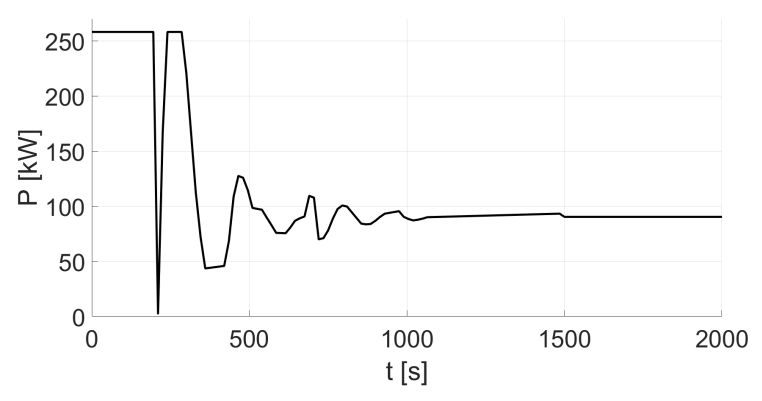

Fig. 6: Total power of the gas burners.

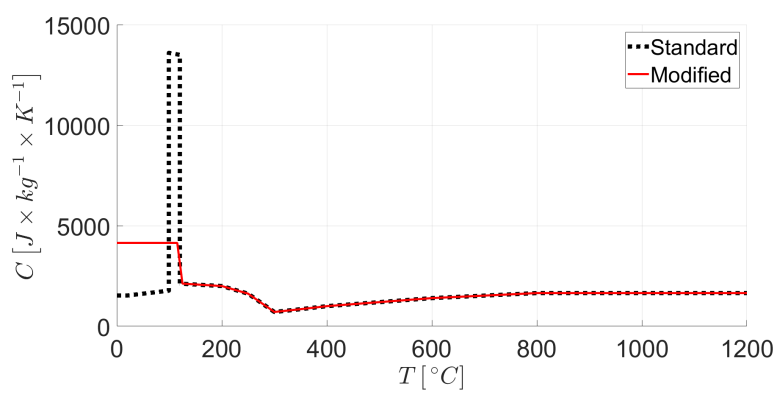

Fig. 8: Timber capacity.

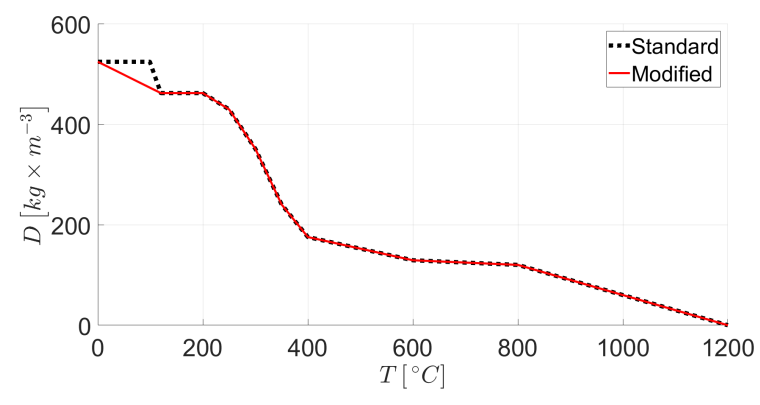

Fig. 7: Timber density.

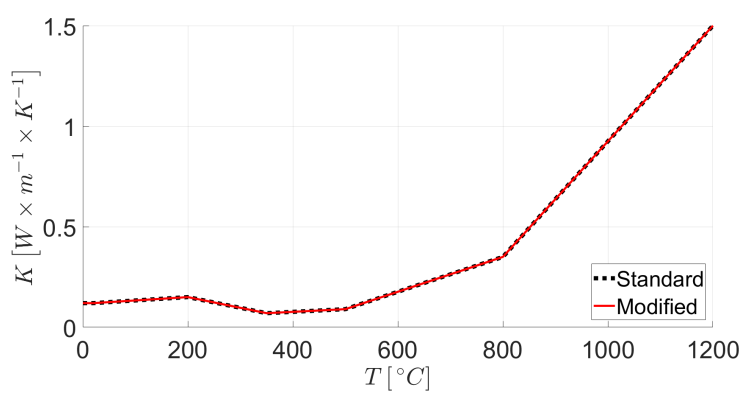

Fig. 9: Timber conductivity.

\subsection{Experiment}

The experiment was realized by fire laboratory of PAVUS a.s. The furnace was heated by 8 natural gas burners. Flue gas exhaust system was performed using a frequency fan placed in a conduit which was connected to the opening $(500 \mathrm{~mm} \times 800 \mathrm{~mm})$ in the floor of the furnace.

The measurement of the temperatures inside the beam was not realized properly, see Fig. 4, most of the sensor wires were installed through the closest surface, thus the temperatures were affected by the cable conductivity.

\subsection{Computations}

The FDS task is composed of one overall mesh consisting of $18 \times 18 \times 12$ elements and three meshes around the beams, each consisting of $4 \times 24 \times 8$ elements. The mesh densities were designed in order to complete the computation approximately in 24 hours.

In OOFEM we modelled a section through the third beam, its domain size is $0.12 \mathrm{~m} \times 0.1 \mathrm{~m} \times 0.32 \mathrm{~m}$. The regular mesh is composed by $24 \times 1 \times 64$ elements, as displayed in Fig. 5. We used brick elements with quadratic approximation. The adiabatic surface temperatures from points around the beam were exported from FDS in periods of $2 s$, but the computational step length in OOFEM had to be set to $0.5 s$ due to numerical stability.

\subsection{Results}

The temperatures were measured in beam number 3 in the section A-A'. We printed the values of the experimental and computed temperatures in points 1, 2, 3, 4, according to Fig. 3. Figs. 10 and 11 present the best results, because the measurement in points 1 and 2 was least affected by the sensor wires. We can see a good congruency of these temperatures. However, the influence of cable conductivity on temperatures in points number 3 and 4 is not negligible. In the results from other sensors the influence of wires was dominant, thus we do not even present them. 


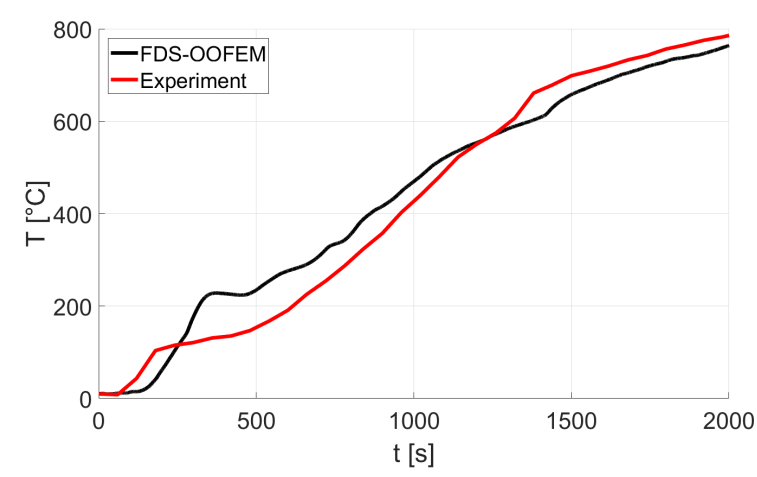

Fig. 10: Temperatures in point number 1.

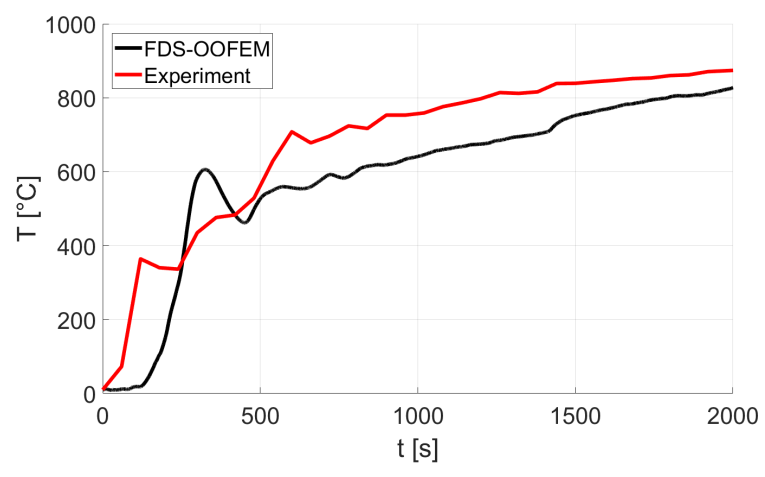

Fig. 12: Temperatures in point number 3.

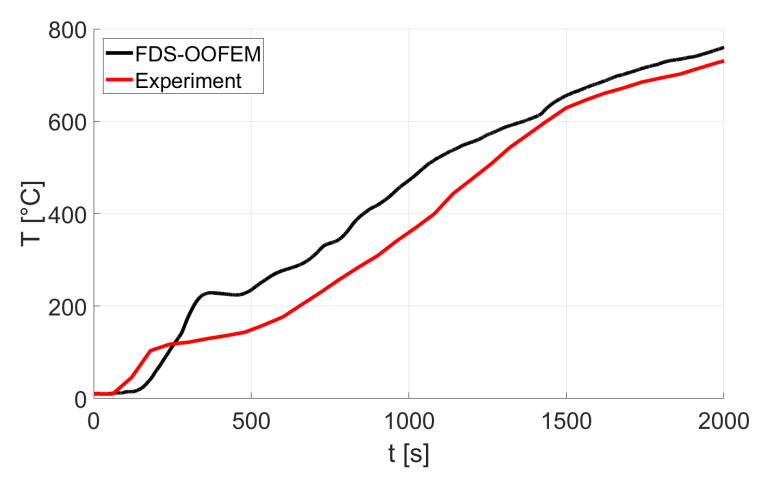

Fig. 11: Temperatures in point number 2.

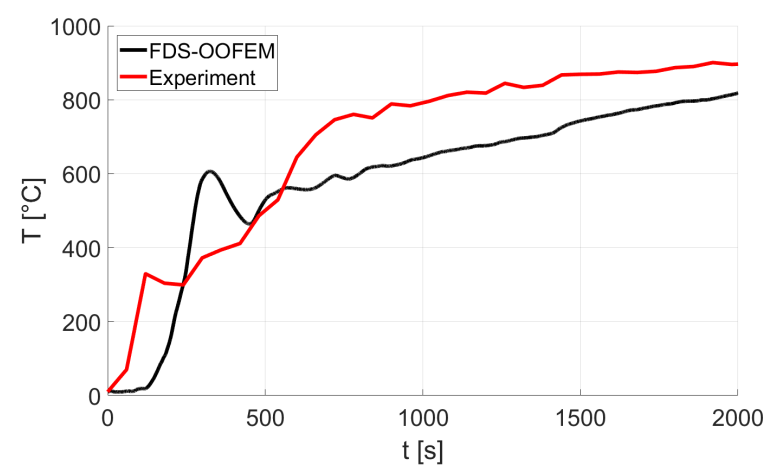

Fig. 13: Temperatures in point number 4.

\section{Conclusions}

Although timber is quite complicated material for predicting its behaviour during combustion, the validation confirms satisfying accuracy of this software tool and its computation based on standard material properties given in EN 1995-1-2. However, we have to make some additional validations on temperatures from bigger depth of a timber element.

In near future we will focus on validation on concrete (thermal response) and steel (thermo-mechanical response) elements. We also consider developing model of the timber material, which could go beyond the limit of 30 minutes by explicit deleting of burnt elements and moving the boundary conditions.

\section{Acknowledgments}

We gratefully acknowledge the financial support from the Czech Technical University in Prague, the grant SGS18/037/OHK1/1T/11

We also gratefully acknowledge the financial support from the Czech Science Foundation, the grant GACR 16-18448S.

\section{References}

Cábová, K., Lišková, N., Novotná, P., Benýšek, M., Zeman, F. and Wald, F. (2017) Modelling of Standard Fire Test. In: Engineering Mechanics 2017, Brno University of Technology, Brno, pp. 226-229.

NIST (2018), FDS - Fire Dynamics Simulator, URL http: / /www.pages .nist.gov/fds-smv/.

Patzák, B. (2018) OOFEM - an object-oriented simulation tool for advanced modeling of materials and structures, URL http: //www. oofem.org/.

Patzák, B., Šmilauer, V., Šmilauer, V., Horák, M. and Pacquaunt, G. (2018) Multi-Physics Integration FrameworkMuPIF 2.0, URL http://mupif.org.

Šulc, S., Šmilauer, Š. and Wald, F. (2017) Coupled Simulation for Fire-Exposed Structures using CFD and ThermoMechanical Models. In: NMM 2017 - Nano \& Macro Mechanics 2017, CTU in Prague, Prague, pp. 121-124. 